\title{
Digital synchronous collaboration workspace and 3D interactions for an AEC project. Decision-making scenario evaluation
}

\author{
Veronika Bolshakova ${ }^{1,2}$ Gilles Halin ${ }^{1,3}$ Pascal Humbert ${ }^{1,4}$ Conrad Boton ${ }^{5}$ \\ ${ }^{1}$ MAP-CRAI - Centre de Recherche en Architecture et Ingénierie UMR n³495 Modèles et \\ simulations pour l'Architecture et le Patrimoine, ${ }^{2}$ CNRS - Centre National de la recherche \\ scientifique, ${ }^{3}$ Université de Lorraine, Nancy, France ${ }^{4}$ ENSAN - Ecole Nationale Supérieure \\ d'Architecture de Nancy, France ${ }^{5}$ École de Technologie Supérieure, Montréal, Canada \\ \{bolshakova; halin; humbert\}@crai.archi.fr \\ Conrad.boton@etsmtl.ca
}

\begin{abstract}
The success of a digitally developed architecture project requires a new vision of collaboration and design practices and the adaptation of the current ones. The recent innovative tools and media extend the design capabilities, and foster the proposal of complex architectural solutions.

This paper aims to evaluate the existing interactive synchronous collaboration technologies and solutions. The evaluation must reveal their potential advantages and future uses for professionals and education in the architecture, engineering, and construction (AEC) industry are assessed. Furthermore, suggestions are made on how to improve the current synchronous collaboration practices in decision-making sessions. Such suggestions consider the complexity of 3D-based project and the multidisciplinary of a project team.
\end{abstract}

The paper introduces a summary of the current digital AEC practices and identifies digital synchronous collaboration requirements for an efficient decision-making session through observation and evaluation of several collaborative session experiments.

Keywords: Computer-supported collaborative work, Architecture, engineering and construction, Multi-user interface, Natural interaction, Decision-making session, Building Information Modeling, 3D model.

\section{$1 \quad$ Introduction}

AEC project development practices have centuries of history, but recent decades have brought a digital-age challenge into the field. Since the project development was transferred not only to a digital sheet but also, to a virtual 3D model of the building, the classic practices of the field had to evolve [1]. Thus, the new tools must resolve the old tasks, and the new aims cannot be achieved from the usual approach angles.

This paper is based on an evaluation of AEC collaborative practices and on experiment observations. It provides the basis for further development of digital synchronous collaboration tools and methods for the Building Information Modeling 
(BIM) projects (at the international research project 4D Collab). Identifying the efficiency factors is essential for a proposal of collaborative work optimization, and is also a key for the digital collaboration protocol definition. The solution must foster natural interface usages and the use of a BIM project model as main support for a decision-making collaborative session (CS).

\section{Synchronous Collaborative at AEC}

\subsection{Digital age and BIM in AEC}

A transformation of conception process will bring a change to the work habits, as well as an adaptation of work methodology to a modernized toolkit. The generation of pioneers in digital architecture has prepared some theoretical and practical basis through many experiments [1]. However, the current generation suffers from a lack of digital tool practices methodology. Yet, the extended 3D models and BIM practices have been integrated into the project development by certain AEC professionals.

Nowadays, many of the AEC professionals develop their projects with the help of the digital tools and practices. But not all of them have yet fully integrated, into their project development and design, the work with 3D semantic enriched models, which is fundamental for the BIM project management and development methods [1, 2] — « The building information model is a three-dimensional geometric model that is data rich» [3]. Though, the AEC projects developed with the innovative digital practices of $3 \mathrm{D}$ modeling reveal the advantages of the improved communication, coordination, and production on a project due to the possibility of interacting with the 3D model content [4]. Such an approach adds a supplementary model components and information annotations to the geometry visualization [5].

The complexity of project model development engages intense collaboration of all AEC professionals; they should find their own new efficient ways of cooperation on a project. Such a modernization of the current practices aims to simplify the work methods and to improve the quality of the project. But as with any innovation, this takes time before complete integration into professional practices, due to the appropriation period. So, the next important step towards BIM-oriented work methods and progressive project management requires us to question the collaborative practices of the AEC project design [6]. The projects conducted with the integration of these practices benefit from improvement in the development coordination inside the project as well as in the communication around the project. These new aspects increase the project complexity. Such a complexity leads to efficient method development for collaboration and exchanges, for a better representation and visualization, for an intense discussion and for more participative decision making.

\subsection{Collaborative Development of an AEC project basis}

Every collaboration approach values the specific features, which correspond to the needs of the work process. For an AEC decision-making a synchronous collaboration has traditionally been the most relevant approach of the interactions on a project [7]. 
The AEC project collaboration hinges on project content. The project scale (a small residential, a large public building, or an urban project) and the development phase (concept, design development, technical design, construction) will together define the development strategy [3]. And such a connection was summarized as a BIM sociotechnical system [8] (Fig.2), where a project technical core (3D CAD, Intelligent Models, Information Management) prepares a project data and work tools for the collaborative manipulations. So, the project progress relies on this system, where the synchronous collaboration plays the role of the first social base to operate the project and creates a work field for further larger scale interactions.
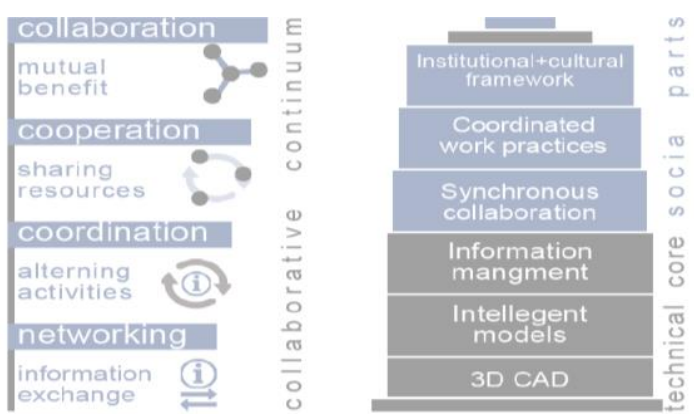

Fig.2 (left) Collaborative continuum concept according to A.Himmelmann [7]

(right) a BIM sociotechnical system.

The social part of the AEC sociotechnical system coordinates with the top of the collaborative continuum concept [9] (Fig.2), because the representation is based on a level system, where the evolution and the complexity grow with the levels along the continuum, as the complexity increases in a sociotechnical system. There are greater levels of trust, time and committed effort in the relationship progressing through the collaborative continuum, which means that every core must fulfill its purpose.

\subsection{Collaborative workspace experiments}

Collaboration engages all the users to share the same environment, to bring the same vocabulary to a work process and to unite project information [7, 10]. Many research works have been dedicated to developing Computer-Supported Collaborative Work (CSCW) scenarios and environments for AEC and design, therefore various collaborative solutions experiments were performed to reveal potential usage: a 3D virtual workspace environment, a holodesk with direct 3D interactions on a seethrough display, life-size 2D sketch representations and hybrid ideation space with an even more immersive environment [11-14]. The CSCW session analysis reveals an importance of a quality interaction (gesturing, navigation, annotation, viewing [15]).

\section{Collaborative experiment summary}

A synchronous collaborative workspace must ensure the physical workspace comfort, along with the uniformity and usability of the virtual workspace $[1,14,15]$, which provides ergonomic interactions with the session workflow. 


\section{$3.1 \quad$ Protocol}

An efficient collaboration relies on the pertinence and the quality of the equipment set, of the interactions and of the collaborative approach choice. A specific protocol organizes the CS. Beforehand the equipment set puts the physical space in order: room measurements and insulation, distances between the equipment and collaborator spaces, as well as, accesses and circulation. Afterwards the equipment setup creates a virtual workspace environment by virtue of the hardware and software installation.

The protocol also includes a collaborative scenario, determined by the CS main objective and interaction requirements. Every CS has an established checklist (equipment, data, users) and corresponding scenarios for a preparation phase (data gathering, scheduling), for the collaboration phase and for the session feedback phase, since every session passes through these phases. Every preparation phase starts from a CS objective, project data, and participants' definition, and then launches a scenario choice (equipment, interactions, roles, etc.). Nevertheless, a protocol leaves a space for an improvisation during the CS. Through the collaboration phase users work with the project data: visualize, manipulate, and annotate. Annotations create a new data and a feedback on the project development.

\subsection{Objective}

The performed experimental sessions have primarily targeted an identification of possible uses for the digital synchronous collaboration protocols on an AEC project. And at the same time, they also have quest for the digital collaborative method advantages and limits, for uses in the AEC industry. The final objective is the specification for efficient new equipment and better protocols.

\subsection{Equipment description}

The decision-making scenario was experimented at the Digital Synchronous Collaboration Workspace (DSCW) of the research center MAP-CRAI (Fig.1). The DSCW associates two digital touch screens, a horizontal one called "The table" (98 inch), and a vertical one "The wall" (46 inch). Both displays are plugged to computers and use a collaborative software "Shariiing" (by Immersion) for the CS experiment.

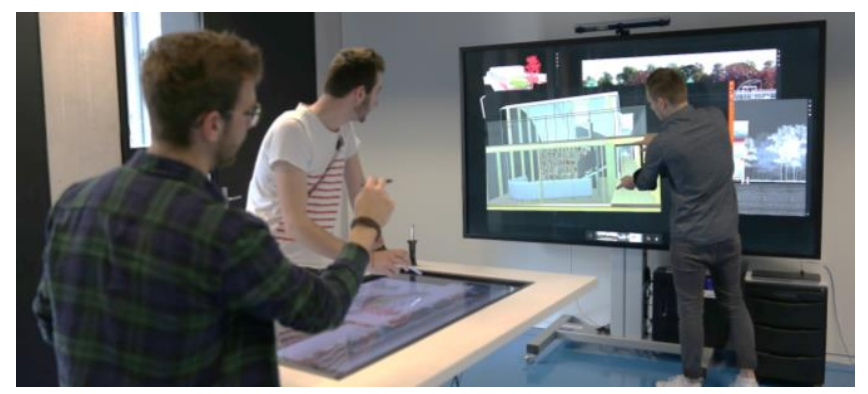

Fig. 1. Design development decision-making session at DSCW of MAP-CRAI, 2016 
The Shariiing software creates a unified digital environment for the project workflow (images, textured 3D models, web pages, pdf files, txt files, etc.) and for homogeneous interactions (upload, visualization, manipulation, annotation, browsing and sharing). The annotation tool functions as a digital pen, so the annotations are mainly sketches or notes. The annotated document is always saved as a new 2D image, so there is no modification made to the original digital 3D model; however, this means that it cannot operate enriched BIM models.

\subsection{Content for a decision-making session}

A design development scenario was investigated at the AEC project decisionmaking session, with a renovation of residential tower as CS subject (Kennedy tower, Nancy, France). The AEC project at a design development stage [3] is typical for a creative development activities. The goal of the session was to design renovation solutions of an exterior facade and an interior entrance hall.

All the participants work in the AEC field, and DSCW experiments gathered them around the subject as users-interactors at the DSCW with a role on a project (architect, client, BIM manager, engineers). The following 2D documents, typical for an AEC project workflow, were uploaded to Shariiing: urban master plan image; facades photos; entrance hall interior photos; the tower master plan, ground floor plan; typical floor plan; local urban development plan text document. In addition, and as a main subject of the study, the 3D digital model of the building with its neighborhood context (BIM NV3 [16]) was suggested by the experiment as a main collaborative interactions base, due to the fact that such a model contains all the information to ensure the creative design development without additional sources.
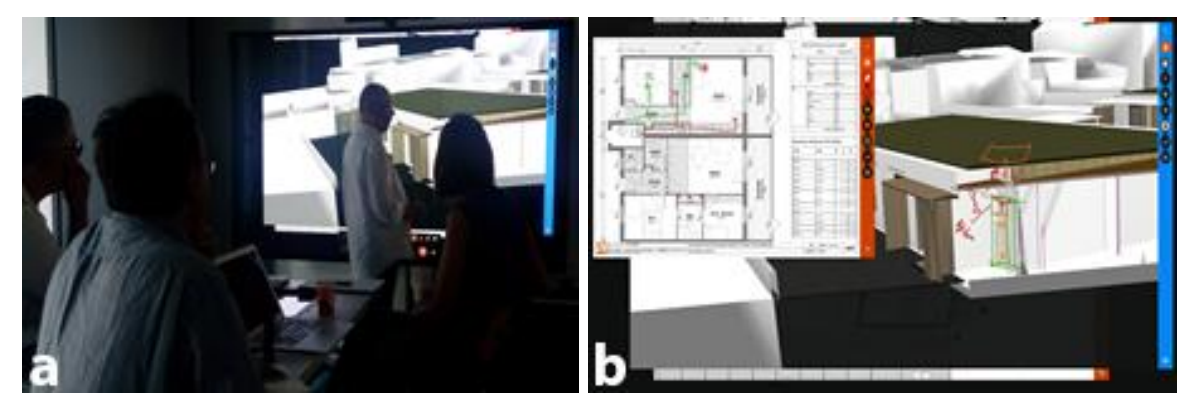

Fig.3 (a) Value engineering session, ongoing AEC project of Mil Lieux agency; (b) Annotations on a 3D model and a floor plan, Mil Lieux agency.

CS proceeded in a following way: informal part; aim and contents by a collaboration manager for the participants; then a problem-solving part - problem visualization, annotation of the existing documents through creative sketching, interprofessional discussion, solution visualization and evaluation; followed by a summary and further definition of development tasks, with an informal farewell at the end. After the first task, users achieve the next one with a higher level of confidence. 
A value engineering scenario was also surveyed at the CS, with the ongoing project of an architecture agency Mil Lieux, in Nancy, France. The decision-making was on a design development project stage with a client, a BIM manager, engineers, and an architect at CS. The goal of the CS was to find a solution for the heating equipment repositioning and for the building borders on unit (Fig. 3).

\subsection{Interaction observation}

The interaction during the CS was observed from four main perspectives: visualization, manipulation, annotation, and coordination.

Visualization. The first step was always the overview of the CS content. The document quality and the manipulation ergonomics defined the choice of a visualized document as a work base, and not the relevance or complexity of the represented information about the project on a document. After a while simultaneous visualization of the same document in a separate window for every user was replaced by a solo document visualization on a larger support surface with an only one person in charge.

Manipulation. Operation, control and management manipulation types were performed. The choice of a document depends directly on the architect's (or other session-leading professional) habits and working methods. A scale change and detail zoom manipulation appeared as a strong argumentation support. The comparison of document versions was another major kind of manipulation. Even with an annotation as the main activity result, users tended still to focus on a verbal discussion to prove their point rather than to explain an idea by sketching or annotation.

Annotation. The annotations are an outcome from the creation or decision-making interactions (Fig.4). Users were often focused only on a 2D annotation of one document, mostly on a 3D virtual model. A creative sketching type of manipulation was most commonly used on the pictures of the project, and on the 3D model fixed views. Meanwhile, an independent sketch drawings and text notes have been used only once. AEC typical procedure was the most efficient: two types of documents were annotated simultaneously - the 3D model fixed point of view and a floor plan. The annotations appear mostly to complete the sketched idea rather than to define a task or a problem.

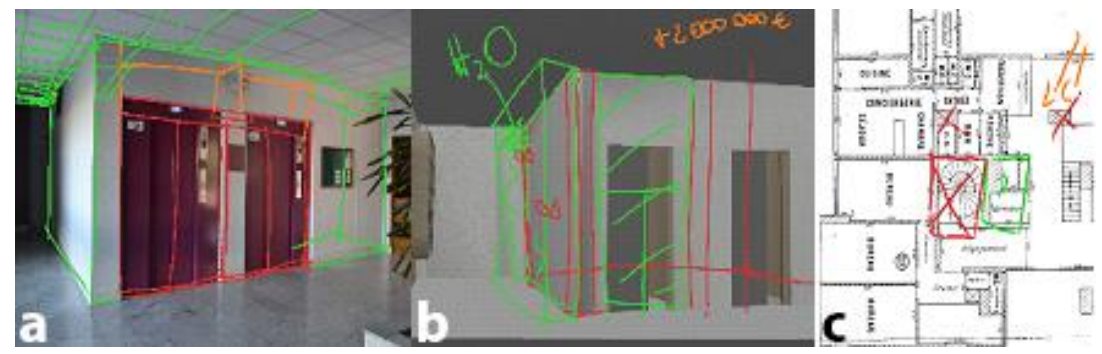

Fig.4 Design development session: a. interior hall photo, b. 3D model, c. floor plan. 
Coordination. Contrary to the professional fixed roles distribution, the CS roles are not fixed. The guiding session role is not permanent, and it passes from one user to another through the development of ideas and interventions, which indicates a certain level of interaction fluency. Experienced professionals from the field had no difficulty in adapting themselves to a new CS method, and were taking less time to achieve the task than their less experienced colleagues.

\subsection{Interpretation and discussion}

The study highlights some notable advantages of the DSCW, but also some challenges to overcome in order to fully benefit from its potential.

Advantages. Efficient decision-making CS are possible with the existing setups of the DSCW. On the equipment level: the most prominent advantages are the quality of the document display, simultaneous visualization, and multi-user interactions. On a decision-making support level: the possibility of 3D digital model interaction enables a very complex level of project management. And on a coordination level: an additional value is the unification of all the document types within the same work environment which follow the same logic.

Limitations. On the equipment aspects: a certain technical improvement and tool development should be undertaken to achieve the addition of new values for the digital synchronous collaboration. The main criticisms are related to the annotation toolkit's lack of instruments, navigation, and ergonomics, and to the CS report creation. The most important problem is the navigation comfort level, users would not interact with the document if it is hard to manipulate. On a decision-making support aspects: A pencil-case, line type choice and the layers have been traditionally a part of the AEC practices and their analog presence is crucial for the DSCW. And the current 3D model use is not yet well adapted for interactions on it. Also, a clear and structured feedback of the CS should be developed. And on a coordination level: Digital synchronous collaboration is relatively new for AEC professionals. DCSW Equipment manipulation and appropriation effort are required for task completion, therefore it takes time to find an efficient mean of expression.

\section{Conclusions}

The digital collaborative workspace technology implementation and current tests by some professionals will raise the future standards, and certainly arouse a wider implementation into the collaborative practices at AEC industry. The DSCW use is simple enough to make the users feel confident in their tool manipulation skills, so the lack of a DSCW use experience has no influence on the collaboration process.

The future work should focus on a technical improvement research: ergonomics, simplicity navigation, additional equipment and instrument development. And also must consider a development of the AEC BIM project collaborative protocols and uses according to the project phase of development (conception, 
technical design, construction) and the project contents, with a full integration of the BIM methods and on a large scale long-term collaborative AEC projects.

\section{Acknowledgments}

This study was developed under the patronage of the Agence Nationale de la Recherche No.ANR-16-CE10-0006-02 (FR) and Fonds National de la Recherche (LU), as a part of the international research project $4 \mathrm{D}$ Collab. We are grateful to the architecture agency Mil Lieux and to all the participants of experiments.

\section{References}

1. Oxman, R.: Digital architecture as a challenge for design pedagogy: theory, knowledge, models and medium. Elsevier 2(29), 99-120 (2008).

2. Delcambre, B.: Rapport de la Mission numérique bâtiment, Ministère du. Logement, de l'Égalité des territoires et de la Ruralité, France (2014).

3. Kensek, K..: Building Information Modeling. 1st edn. Routledge, United Kingdom (2014).

4. Succar, B.: Building Information Modelling: conceptual constructs and performance improvement tools. PhD Thesis, School of Architecture and Built Environment Faculty of Engineering and Built Environment University of Newcastle, Australia (2013).

5. Mazza, R.: Introduction to Information Visualization. Springer-Verlag London,UK (2009).

6. Boton, C., Kubicki, S., Halin, G.: Method to design coordinated multiple views adapted to user's business requirements in $4 \mathrm{D}$ collaborative tools in $\mathrm{AEC}, 15$ th International Conference on Information Visualisation, pp. 96-101. London, United Kingdom (2011).

7. Wilkinson, P.: Construction Collaboration Technologies: The Extranet Evolution. Taylor \& Francis, London (2005)

8. Kennerley, B.: BIM is a Sociotechnical System. WSP Group plc., http://www.wspgroup.com/en/wsp-group-bim/BIM-home-wsp/what-is-bim/, 2017/02/20

9. Himmelman, A.: Collaboration for a change: Definitions, decision-making models, roles, and collaboration process guide, Himmelman Consulting, Minneapolis (2004).

10. Achten, H.H.: Requirements for Collaborative Design in Architecture. In: Timmermans, H.J.P, Vries, B. (eds.) SIXTH DESIGN AND DECISION SUPPORT SYSTEMS IN ARCHITECTURE AND URBAN PLANNING, Part one: Architecture Proceedings Avegoor, pp. 1-13. Eindhoven: Technische Universitet Endhoven, the Netherlands (2002).

11. Achten, H.: Futures scenario for a collaborative design session and feature list. In: Stellingwerff, M., Verbeke, J. (eds.) Accolade - Architecture. Collaboration. Design., pp. 163-167. Delft University Press, Delft, the Netherlands (2001).

12. Hilliges, O., Kim, D., Izadi, S., Weiss, M., Wilson, A.D.: HoloDesk: Direct 3D Interactions with a Situated See-Through Display. In: SIGCHI Conference on Human Factors in Computing Systems, CHI 2012, pp. 2421-2430. ACM New York, USA (2012).

13. Dorta, T., Kalay, Y.: Comparing Immersion in Collaborative Ideation through Design Conversations, Workload and Experience. In: Integration Through Computation. Presented at the ACADIA, pp. 216-225. Bnaff, Canada (2011).

14. Dorta, T., Kinayoglu, G.: Towards a new representational ecosystem for the design studio. In: Rethinking Comprehensive Design: Speculative Counterculture, The 19th International Conference on Computer-Aided Architectural Design Research in Asia, pp. 699-708. Kyoto: Kyoto Institute of Technology (2014).

15. Tory, M., Staub-French, S.: Physical and Digital Artifact-Mediated Coordination in Building Design. Computer Supported Coop Work, 4(17), pp 311-351. Springer (2008).

16. "Le Moniteur - Cahier pratique", Cahier détaché n2, no. 5763, pp. 44, (2014). 